\title{
Un modèle révisé de propagation électromagnétique dans les sols pour l'étude de réseaux sans fil de capteurs souterrains
}

\author{
Frank Kataka BANASEKA ${ }^{1,2}$, Hervé FRANKLIN ${ }^{2}$, Ferdinand KATSRIKU ${ }^{1}$, \\ Jamal-Deen ABDULAI ${ }^{1}$, Akon EKPEZU ${ }^{1}$, Isaac WIAFE ${ }^{1}$ \\ 1. Department of Computer Science, University of Ghana, Legon, Ghana. \\ fkatsriku@gmail.com \\ 2. Laboratoire Ondes et Milieux Complexes, Unité mixte de recherche du CNRS 6294, \\ Université Le Havre Normandie, France \\ herve.franklin@univ-lehavre.fr
}

\section{Résumé :}

Ces dernières années, il y a eu un vif intérêt pour la recherche sur l'Internet des objets connectés sous terre et avec cela, la nécessité de bien comprendre et caractériser l'environnement d'exploitation, ce dernier dépendant étroitement de la fréquence utilisée par rapport à la taille des inhomogénéités locales. Dans cet article, un modèle est proposé, basé sur le Principe de Peplinski pour la propagation des ondes dans les sols qui prend en compte les pertes imputables à la présence des inhomogénéités locales. On suppose que les inhomogénéités sont des obstacles tels que des pierres ou cailloux, de taille modérée, tous identiques et répartis aléatoirement dans l'espace. Un nouveau nombre d'onde est obtenu grâce à une combinaison de la théorie de la diffusion multiple et du principe de Peplinski. Étant donné que ce dernier considère propagation dans un milieu homogène (sans obstacles), le nombre d'onde qu'il fournit est pris en compte dans celui résultant de la théorie de la diffusion multiple. Le nombre d'onde effectif ainsi obtenu est comparé numériquement à celui de Peplinski seul d'une part puis avec celui de la diffusion multiple seul d'autre part. Les résultats obtenus suggèrent que le modèle proposé fournit une meilleure précision dans l'estimation du nombre d'onde que les schémas utilisés auparavant.

Mots-clés :

Internet des objets souterrains, Diffusion multiple dans le sol, Pertes, Réseaux de capteurs sans fil.

\section{Introduction}

Les réseaux de capteurs souterrains sans fil (WUSN) sont un domaine émergent qui a attiré l'attention de nombreux des chercheurs. En effet, les WUSN ouvrent de nouvelles possibilités pour la surveillance souterraine (application en agriculture) et la communication. Ces WUSN fonctionnent par émission-réception d'ondes électromagnétiques (EM) et sont maintenant interconnectés pour former "l'Internet des Objets Souterrains (IoUT)" précieux pour la prise de décision et le contrôle. 


\section{Thème 3 - Instrumentation, mesures, imagerie et télédétection}

L'atténuation des ondes EM dans le sol est beaucoup plus élevée que dans l'air. Cela a un effet limitant sur la qualité de la liaison. Des obstacles tels que des pierres ou des rochers provoquent la diffusion des ondes dans tout l'environnement souterrain. De plus, une augmentation de la portée de communication mais aussi de la teneur volumétrique en eau du sol en raison de l'irrigation ou de la pluie et la nature du milieu, notamment la teneur en argile impactent l'atténuation du champ EM propagé et entraînent des pertes élevées du signal. Pour caractériser efficacement l'environnement de propagation, la mise au point de modèles précis et robustes est nécessaire. Différents modèles ont été proposés dans la littérature pour l'étude de la propagation des ondes EM dans le sol (PEPLINSKI et al., 1995 ; VURAN \& AKYILDIZ, 2010).

À notre connaissance, les modèles d'atténuation qui prennent en compte la diffusion multiple par des objets enfouis doivent être développés. Dans cet article, sur la base de résultats existants (VURAN \& AKYILDIZ, 2010) où une relation pour les pertes est proposée, nous considérons le problème de la propagation des ondes EM (TSANG et al., 2001) dans un sol contenant des diffuseurs. Un modèle du nombre d'ondes effectif est présenté qui tient compte de l'absorption due à la permittivité diélectrique et de la diffusion multiple. Les constantes de propagation dérivées de ce nombre d'onde complexe peuvent être utilisées ensuite pour le calcul de l'affaiblissement du signal. Les résultats sont comparés à ceux précédemment rapportés dans la littérature. De plus, une étude paramétrique est également réalisée qui montre les effets de la concentration des obstacles ou de la teneur volumétrique en eau sur l'atténuation du signal.

\section{Absorption due à la permittivité diélectrique des sols}

Dans ce qui suit on considère une distribution aléatoire uniforme de sphères diélectriques de rayons $a$ et de permittivité diélectrique relative $\varepsilon_{p}$ - par rapport au vide de permittivité diélectrique $\varepsilon_{0}$ - placées dans un milieu (sol) de permittivité diélectrique $\varepsilon$. Soit $n_{0}$ le nombre de sphères par unité de volume. Le nombre d'onde effectif $K$ du milieu effectif ainsi constitué est donné par

$K^{2}=k^{2}\left(1+\frac{3 c y}{1-c y}\right)$

où $k=\omega \sqrt{\mu \varepsilon}$ est le nombre d'onde dans le sol en l'absence de diffuseurs, $y=\left(\varepsilon_{p}-1\right) /\left(\varepsilon_{p}+2\right)$ et $c=n_{0} v_{0}$ avec $v_{0}=4 \pi a^{3} / 3$. L'existence d'une partie imaginaire pour $K$ traduit la présence du phénomène d'absorption dans le milieu lors de la propagation. Dans l'équation (1), la permittivité diélectrique $\varepsilon$ peut être celle dérivée du principe de Peplinski qui est discuté plus loin. 


\section{XVIèmes Journées Nationales Génie Côtier - Génie Civil \\ Le Havre 2020}

\section{Atténuation des ondes due à la diffusion multiple dans le sol. Approximation Quasi Cristalline (QCA) pour les milieux denses}

Les phénomènes de diffusion multiple se produisent dans le sol en présence d'inhomogénéités ou d'obstacles distribués de façon aléatoire. L'équation (1) ne prend, en aucun cas, en considération la diffusion multiple des ondes par les sphères de rayon a. Il paraît, dans ces conditions, approprié d'y ajouter un terme tenant compte de cette diffusion multiple.

Pour résoudre le problème de la propagation des ondes dans les sols, il est utile de simplifier le problème en premier lieu. Les simulations de diffusion peuvent être effectuées sur un volume d'essai contenant un grand nombre de sphères mais formant en même temps une petite partie (le volume élémentaire représentatif) de l'ensemble du système.

Un sol contient normalement des diffuseurs de formes, tailles et matériaux variés (milieu poly dispersé). Dans ce travail, par souci de simplicité, on suppose que tous les diffuseurs sont identiques (milieu mono dispersé). L'approximation quasi-cristalline (QCA) et le formalisme de la matrice T ont été utilisés (TSANG et al., 2001). Dans la QCA, une moyenne conditionnelle sur les positions des sphères est effectuée. Les détails techniques du calcul menant à la formule du nombre d'onde effectif sont hors du cadre du présent travail. Notons seulement que dans le contexte de la QCA et pour les faibles concentrations de diffuseurs $(c<0.4)$ et du produit $k a(<0.5)$, le nombre d'onde $K$ est tel que

$$
K^{2}=k^{2}\left(1+\frac{3 c y}{1-c y}\left[1+i \frac{2 k^{3} a^{3}}{3} \frac{y}{1-c y} \frac{(1-c)^{4}}{(1+2 c)^{2}}\right]\right) \text {. }
$$

La plupart des cartes de capteurs souterrains sans fil telles que les MICA2 sont conçues pour fonctionner dans une plage de 300 à $400 \mathrm{MHz}$. Idéalement, des fréquences de fonctionnement de 300 à $900 \mathrm{MHz}$ sont recherchées. Cela garantit que les capteurs restent discrets, une propriété qui est particulièrement utile pour les applications de sécurité. A une fréquence de $433 \mathrm{MHz}$, la condition $k a>0,1$ est vérifiée si les sphères ont un rayon a $>1,1 \mathrm{~cm}$ (ceci entraîne $n_{0}<33873 \mathrm{~m}^{-3}$ pour une concentration $c=0.2$ par exemple). Noter qu'à une fréquence de $868 \mathrm{MHz}$, la condition $k a>0,1$ est vérifiée si $a>0.55 \mathrm{~cm}$. La figure 1 montre les variations de la vitesse de phase normalisée $\operatorname{Re}(k / K)$ et de la tangente de perte $2 \operatorname{Im} K / \operatorname{Re} K$ en fonction de la concentration. Elles sont obtenues à partir de la formule (2) pour trois valeurs du produit (sans dimension) $k a$. La permittivité diélectrique du sol est $\varepsilon=8,854 \times 10^{-12} \mathrm{~F} \mathrm{~m}^{-1}$ tandis que la permittivité relative des sphères est $\varepsilon p=3,2$. Lorsque la concentration $c$ augmente, la vitesse de phase diminue de façon monotone tandis que la tangente de perte croît initialement jusqu'à une valeur maximale puis se met à décroître. 

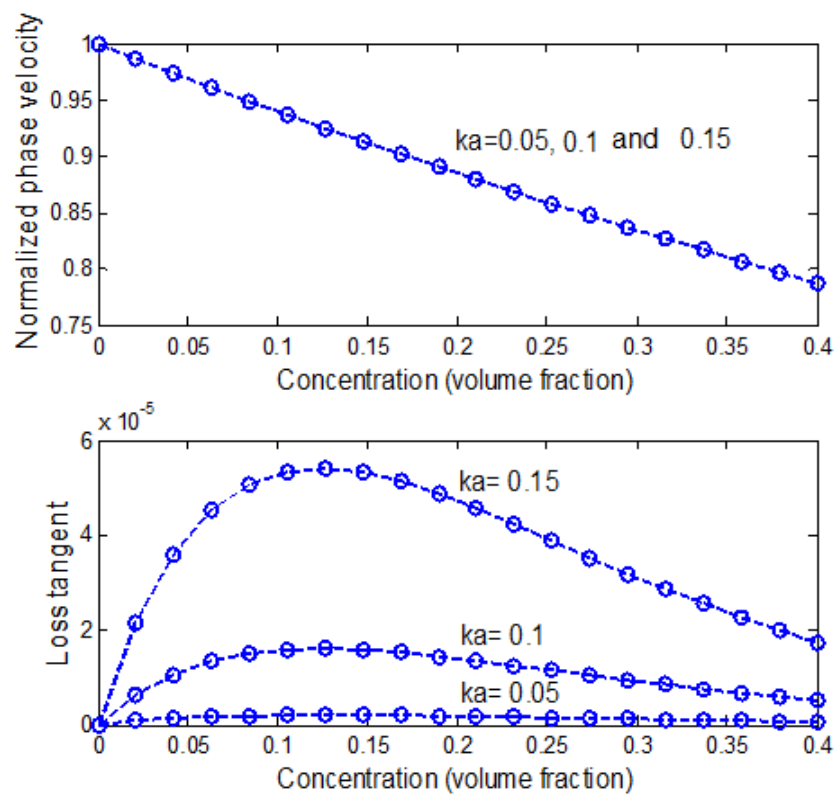

Figure 1. Vitesse de phase normalisée et tangente de perte en fonction de la concentration.

\section{Prise en compte du modèle de Peplinski}

Un modèle diélectrique semi-empirique pour les sols, couvrant la gamme de fréquence 0,3-1,3 GHz a été proposé (PEPLINSKI et al., 1995). Le modèle fournit des expressions pour les parties réelles et imaginaires de la constante diélectrique relative d'un sol en termes de composition texturale (fractions de sable, de limon et d'argile), de densité apparente et de teneur en humidité du sol. Une comparaison de résultats expérimentaux mesurés avec les prédictions basées sur le modèle semi-empirique montre que ce dernier sous-estime la partie réelle de la constante diélectrique dans les cas où la teneur en eau du sol est élevée. Il sous-estime également la partie imaginaire de la constante diélectrique pour tous les types de sols et la teneur en humidité.

En supposant une permittivité diélectrique complexe $\varepsilon=\varepsilon^{\prime}-i \varepsilon^{\prime \prime}$ pour le sol, où $\varepsilon^{\prime}$ et $\varepsilon^{\prime \prime}$ sont des quantités réelles détaillées dans LIEDMANN et al. (2018), le nombre d'onde complexe s'écrit :

$\gamma=\varsigma+i \varpi$

où

$$
\begin{gathered}
\varsigma=\operatorname{Re}(\gamma)=\omega \sqrt{\frac{\mu \varepsilon^{\prime}}{2}\left[\sqrt{1+\left(\frac{\varepsilon^{\prime \prime}}{\varepsilon^{\prime}}\right)^{2}}-1\right]}, \\
\varpi=\operatorname{Im}(\gamma)=\omega \sqrt{\frac{\mu \varepsilon^{\prime}}{2}\left[\sqrt{1+\left(\frac{\varepsilon^{\prime \prime}}{\varepsilon^{\prime}}\right)^{2}}+1\right]} .
\end{gathered}
$$




\section{XVI'̀mes Journées Nationales Génie Côtier - Génie Civil \\ Le Havre 2020}
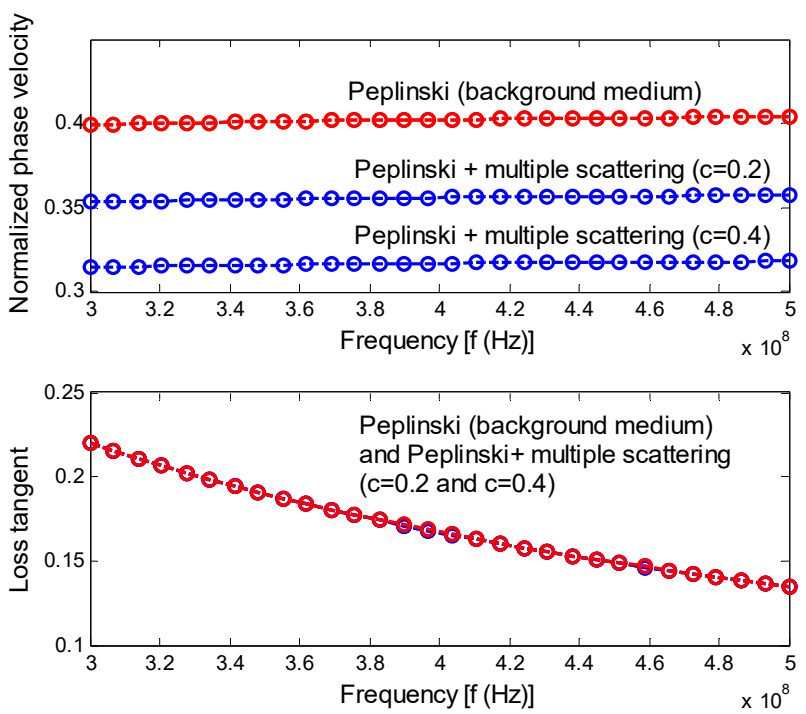

Figure 2. Vitesse de phase normalisée et tangente de perte en fonction de la fréquence pour une teneur en eau de $5 \%$.
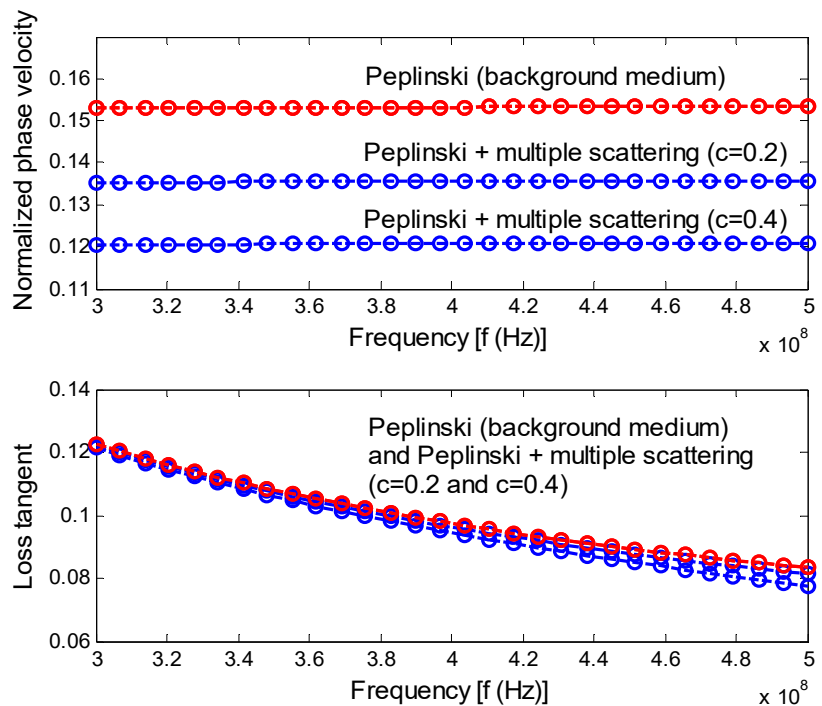

Figure 3. Vitesse de phase normalisée et tangente de perte en fonction de la fréquence pour une teneur en eau de $50 \%$.

Les courbes rouges des figures 2 et 3 montrent les résultats obtenus en utilisant le principe de Peplinski. Les courbes bleues montrent les résultats obtenus lorsque, à la fois, le modèle de Peplinski (sol homogène) et la diffusion multiple des sphères sont pris en compte. Le rayon considéré pour les sphères est $a=1,10 \mathrm{~cm}$ pour une concentration $c=$ 0,2 puis $c=0,4$. La figure 2 montre les graphes de la vitesse de phase et de la tangente de perte en fonction de la fréquence lorsque la permittivité diélectrique du sol est donnée par l'équation (3) avec 5\% de teneur en humidité. Ce nombre d'onde est introduit dans l'équation (10) pour donner le nombre d'ondes qui combine le modèle de Peplinski, 


\section{Thème 3 - Instrumentation, mesures, imagerie et télédétection}

l'absorption (sec. 2) et la diffusion multiple (sec. 3). Pour une fréquence fixée, on peut voir que la vitesse diminue au fur et à mesure que la concentration augmente et est beaucoup plus petite que sur la figure 1. Pour la tangente de perte, les courbes bleues et rouges décroissent et montrent la même tendance. La figure 3 correspond au cas où la teneur en humidité est égale à $50 \%$. Pour la tangente de perte, les courbes bleue et rouge bien que montrant la même tendance, s'éloignent lorsque la fréquence augmente. Le rayon considéré pour les sphères est le même que sur la figure 2: Une comparaison des figures 2 et 3 , montre que l'augmentation de la teneur en humidité diminue à la fois la vitesse de phase et la tangente de perte et a, de ce fait, une grande influence sur la propagation des ondes.

\section{Conclusions}

Nous avons comparé le nombre d'onde basé sur un modèle de diffusion multiple dans un sol avec des prédictions basées sur le modèle semi-empirique de Peplinski. Le sol est supposé contenir une distribution aléatoire de diffuseurs, ce qui rend l'estimation de la propagation du signal dans ce milieu assez difficile. En combinant les effets de la diffusion multiple et le principe de Peplinski, nous dérivons un modèle mixte du nombre d'onde effectif dans le sol qui tient compte à la fois de l'humidité et des roches de petite taille (de forme sphérique). Les résultats numériques montrent comment l'intégration de la diffusion multiple dans l'analyse modifie les pertes. Celles-ci sont un paramètre important pour tout système de communication sans fil et pour la conception et la conceptualisation de réseaux de capteurs souterrains sans fil (WUSN). Le modèle est développé en tenant compte du fait que la zone des inhomogénéités se situe suffisamment loin des capteurs (en champ proche les phénomènes non-linéaires inévitables peuvent invalider le modèle).

\section{Références bibliographiques}

LIEDMANN F. H., HOLEWA C., WIETFELD C. (2018). The radio field as a sensor A segmentation based soil moisture sensing approach. IEEE Sensors Applications Symposium, SAS 2018 - Proceedings. https://doi.org/10.1109/SAS.2018.8336755

PEPLINSKI N.R., ULABY F.T., DOBSON M.C. (1995). Dielectric properties of soils in the 0.3-1.3-GHz range. IEEE Transactions on Geoscience and Remote Sensing, Vol. 33(3), pp 803-807. https://doi.org/10.1109/36.387598

TSANG L., KONG J.A., DING K.-H., AO C.O. (2001). Particle positions for dense media. characterizations and simulations. In Scattering of Electromagnetic Waves: Numerical Simulations, 1st ed., New York, Wiley, chap. 8, section 2, pp 403-451. https://doi.org/10.1002/0471224308.ch8

VURAN M.C., AKYILDIZ F. (2010). Channel model and analysis for wireless underground sensor networks in soil medium. Physical Communication, Vol. 3(4) pp 245-254. https://doi.org/10.1016/j.phycom.2010.07.001 